\title{
Inversión publicitaria y pluralismo informativo: una aproximación comparada al caso de la prensa en $\mathrm{Chile}^{1}$
}

\section{Investment in advertising and media pluralism. A comparative approach to the case of the press in Chile}

\author{
Enrique Vergara Leyton \\ Pontificia Universidad Católica de Chile \\ evergaral@uc.cl
}

\author{
Claudio Garrido Peña \\ Universidad Diego Portales \\ claudio.garridop@udp.cl
}

\author{
Abraham Santibáñez Martínez \\ Universidad Diego Portales \\ abe@abe.cl \\ Pedro Pablo Vera Soto \\ Universidad Diego Portales \\ pedroverasoto@gmail.com
}

\begin{abstract}
Resumen
A través de un estudio de caso a empresas anunciantes que se han visto enfrentadas a denuncias que afectan su reputación, este artículo busca explorar la relación entre pluralismo informativo y publicidad, identificando los posibles riesgos que conlleva para el medio su dependencia de la inversión publicitaria. Como resultado de un análisis tanto cuantitativo como cualitativo, se advierte que la influencia del avisador sobre el medio es difusa, no se da de forma directa sino que se asume como algo implícito, de alta complejidad donde intervienen dimensiones políticas, comerciales y de práctica profesional, lo que impacta en la producción de contenidos y en la generación de un espacio de discusión pública.
\end{abstract}

\footnotetext{
1 Este artículo forma parte de las investigaciones "Pluralismo informativo e inversión publicitaria en medios escritos. Un análisis en torno a casos de empresas enfrentadas a situaciones de crisis" y "Pluralismo, construcción de audiencias y propuestas mediáticas. Un estudio comparado", ambas realizadas con el apoyo financiero del Fondo para proyectos Semilla de la Universidad Diego Portales.
} 
Palabras clave: Pluralismo, publicidad, periodismo, opinión pública, información.

\begin{abstract}
Through a case study to advertisers who have been faced with complaints involving its reputation, this article seeks to explore the relationship between pluralism and advertising, identifying possible risks to the Media dependence on advertising investment. From a quantitative and qualitative analysis warns that the influence of the warning on the environment is diffuse, not given directly but assumed as implicit, which involved highly complex political, commercial and professional practice, which has a major impact on content production and generation of a public discussion space.
\end{abstract}

Key Word: Pluralism, journalism, advertising, public opinion, information.

\title{
Introducción
}

¿Hasta qué punto los medios están dispuestos a cuestionar a quienes constituyen su fuente de financiamiento? Esta pregunta planteada por Pérez-Tornero (1991) pone de relieve una dimensión cada vez más compleja de la relación periodismo-publicidad y su impacto en el pluralismo de los medios y ha sido un permanente foco de conflicto y tensiones. No obstante, esta relación ha sido escasamente abordada desde la academia, restando de esta forma profundidad al debate sobre los medios de comunicación y la libertad de expresión en Chile.

Llama la atención, en este sentido, que el debate sobre pluralismo en medios se ha centrado casi exclusivamente en la concentración económica y los supuestos peligros que conlleva este fenómeno, a través del surgimiento de posibles monopolios que amenazan la libre competencia y el pluralismo ${ }^{2}$. Sin pretender desconocer los aportes de esta perspectiva al debate, los efectos de la inversión publicitaria sobre el pluralismo han sido escasamente estudiados. En general, podemos señalar que la reflexión ha sido bastante pobre, por no decir nula. Los estudios sobre periodismo y publicidad se han desarrollado de forma autónoma, sin abordar áreas comunes entre los límites de la actividad periodística y el quehacer publicitario (Santibáñez y Vergara, 2008). Es a partir de esta zona de roces, entre el ejercicio periodístico y publicitario, que el objetivo de este artículo es entregar una aproximación teórica y empírica respecto de los posibles impactos de la inversión publicitaria en el tratamiento informativo y el pluralismo en medios escritos en Chile.

2 A modo de ejemplo de trabajos que abordan la relación entre concentración de la propiedad de los medios y sus posible efectos en el pluralismo informativo, podemos citar los casos de Sánchez-Tabernero (2002); Bustamante (1994); Llorens (2001); Sunkel y Geoffroy, (2001); Corrales y Sandoval (2003) y Portales (1999). 


\section{Contexto de discusión: publicidad y periodismo como referentes de modernidad}

Para Thompson (1998) el desarrollo de los medios de comunicación desde los primeros impresos está íntimamente relacionado con las transformaciones institucionales que han dado forma a la modernidad y constituyen parte integral de la sociedad actual. En consecuencia, si se quiere comprender la naturaleza de la modernidad, esto es, las características institucionales de las sociedades modernas y sus condiciones de vida, se debe otorgar un lugar central a los media. Según Thompson, con el advenimiento de las sociedades modernas, tuvo lugar una transformación cultural sistemática como consecuencia de los nuevos modos de producción y circulación de los bienes simbólicos a una escala sin precedentes, cambiando de forma irreversible las pautas de comunicación e interacción, lo que en un sentido amplio se ha denominado como la "mediatización de la cultura".

En este mismo sentido, Brunner (1995) plantea que los medios presiden el tránsito a la modernidad, ya que contribuyen más que ninguna otra institución a desencadenar el paso de una cultura organizada jerárquicamente a otra distribuida masivamente a partir de un nuevo tipo de relación entre los mercados, las industrias y los bienes culturales.

\subsection{Cómo entender la publicidad en una sociedad de masas}

No es posible aproximarnos a un fenómeno central de la cultura de masas como es la publicidad, si no lo contextualizamos histórica y socialmente. La comprensión de la publicidad conlleva situar la reflexión al menos en dos dimensiones. En primer lugar, a nivel industrial en cuanto recurso básico para dirigir la demanda de productos y servicios en una economía de mercado y como una de las principales fuentes de financiamiento de la industria de medios de comunicación. Por otra parte, la publicidad en cuanto producción de bienes simbólicos, adquiere una dimensión sociocultural de primer orden en la cultura de masas, por su influencia en las visiones de mundo y su capacidad para transmitir parámetros de comportamiento, socialización y representación. Barthes, en sus últimas reflexiones, plantea que la publicidad puede llegar a ser "beneficiosa" y la sitúa entre los "grandes alimentos psíquicos del hombre", junto a la literatura, los deportes y la moda, entre otros (Madrid, 2005, p. 214).

La publicidad es hija de la Revolución Industrial. El desarrollo de grandes centros urbanos, unido a los avances tecnológicos en el ámbito de la imprenta, sentaron las bases para el desarrollo de los primeros soportes publicitarios masivos: el afiche y el aviso en prensa. Como consecuencia de este desarrollo, la comunicación comercial adquirió por primera vez una dimensión de masas y el espacio público urbano se transformó en un espacio de comunicación comercial; en un terreno de signos, al decir de Susan Sontag (2001).

En el caso del afiche, su utilización implicó que se licitaran las paredes de ciudades como Londres y París bajo la concepción de espacio publicitario y, además, introdujo importantes 
cambios en los criterios estéticos al pasar su diseño desde las bellas artes a la comunicación social. El aviso publicitario en prensa significó un aumento en el tiraje y número de páginas, transformando a la prensa en un medio de masas bajo una nueva concepción de audiencia, consolidando de esta forma un nuevo modelo de negocio editorial. Este cambio en el mundo editorial se tradujo en la aparición de los primeros "suplementos" y de medios que nacieron como soportes publicitarios segmentados. Este es el caso, por ejemplo, de las revistas como Vogue, nacida en 1909, donde se promovían productos de calidad asociados a los nuevos estilos de vida de la alta burguesía.

En conclusión, podemos señalar que la publicidad responde a la necesidad de crear nuevos mercados para el consumo de productos cada vez más asequibles -productos de la Revolución Industrial-, transformándose en una necesidad económica para el desarrollo y expansión de la industria de medios, los que, a su vez, se constituyen en un dispositivo central para la consolidación de una opinión pública informada, requisito básico de las democracias modernas. Es decir, la publicidad desde esta perspectiva se entiende como una consecuencia inevitable de la modernidad. Más que crear necesidades, la publicidad responde a las necesidades de la modernidad, a través de su contribución a la expansión de las industrias culturales y, por consiguiente, en la configuración de una opinión pública informada y al desarrollo del mercado de bienes y servicios.

Una de las consecuencias de lo anteriormente señalado, fue el rápido desarrollo y expansión de las agencias de publicidad en Estados Unidos entre los años 1910 y 1929, impulsado por un acelerado crecimiento de su mercado interno: como producto del fuerte incremento en la producción de bienes, se necesitó de estrategias de marketing para activar su consumo. En la etapa de la postguerra (1945-1960), se va a triplicar la inversión publicitaria como consecuencia del aumento explosivo de los bienes de consumo y las innovaciones tecnológicas en el ámbito de los medios de comunicación de masas, como fue la televisión y la internacionalización de la economía de Estados Unidos, especialmente hacia Europa Occidental. A partir de 1960, la expansión de las agencias de publicidad se traslada hacia Asia y América Latina. En efecto, si entre 1915 y 1959 se inauguran 50 oficinas fuera de Estados Unidos, entre 1960 y 1971 se abren 210 (Janus y Rocagliolo, 1987).

En el caso de Chile, la expansión del mercado publicitario se da en la segunda mitad de la década de los 70 del siglo XX, que es cuando, como consecuencia del Golpe de Estado de 1973, se instaura un nuevo modelo económico de libre mercado, donde uno de sus principales desafíos es crear las condiciones socioculturales para la masificación del consumo. Es así como entre los años 1975 y 1980, la inversión publicitaria en Chile crece desde 27 a 299 millones de dólares, es decir, 1.000\% en términos nominales (Catalán, 1982). 


\subsection{Periodismo, espacio público y deliberación}

El periodismo admite al menos dos lecturas respecto de su desarrollo. Por una parte, la dimensión tecnológica asociada al desarrollo de la industria de los medios, entendida esta como una institución central de las sociedades modernas donde la publicidad es fundamental para su desarrollo y expansión, y por otra, como un actor medular de las sociedades democráticas y de las libertades públicas al articular una opinión pública informada y deliberante. En este sentido, Lazarsfeld (1964) en la década de los cuarenta, ya señalaba que la ampliación del debate político más allá de los límites del contacto cara a cara era solo posible gracias a los massmedia. De ahí que diarios, revistas, radios y televisión se tornaran esenciales para el proceso de "dar sentido a la reunión" cuando estas reuniones abarcan a millones de participantes. Por lo tanto, el concepto mismo de opinión pública está íntimamente relacionado al desarrollo de la industria de medios y su consecuencia en el plano cultural: la cultura de masas. Esta relación de dependencia se advierte en la noción de idea de opinión pública propuesta por Lippmann en 1949 en el contexto de una democracia liberal y en el entendido de que democracia es inseparable de participación y esta, de información (Moragas, 1985, p. 17).

No obstante lo anterior, es importante mencionar la postura crítica de Habermas (1981) respecto de la idea de una opinión pública asociada íntimamente a los medios de comunicación de masas. Para este autor, a partir de 1870, que es cuando la esfera pública empieza a estar dominada por un Estado cada vez más fuerte y expansivo, la prensa comienza a perder su independencia al representar intereses económicos. Esta situación es consecuencia de procesos de fusión y del surgimiento de conglomerados y monopolios industriales y mediáticos que transforman profundamente el rol del periodista. Habermas asocia este declive de esfera pública informada al triunfo de lo que él denomina "racionalidad instrumental", la que opera bajo los principios de dinero y poder, recompensa y castigo, a lo cual va a contraponer la noción de "racionalidad comunicativa", que supone un proceso de interacción entre individuos capaces de hablar y actuar, coordinando sus acciones para arribar a un acuerdo, donde el lenguaje y la cultura serían los elementos constitutivos de esta nueva forma de racionalidad.

Si bien Habermas (1987) considera que los medios están sometidos a criterios comerciales, y por lo tanto, no ofrecerían un marco adecuado para la discusión democrática, posteriormente dejaría abierta esta posibilidad.

\subsection{Inversión publicitaria y prensa escrita en Chile}

El año 2011 la inversión publicitaria en medios alcanzó los 659.478 millones de pesos, equivalente a 1.364 millones de dólares (ACHAP, 2012). De este total, el mayor porcentaje se concentró en Televisión Abierta con un 44,9\%, seguido por los Diarios con un 26,6\%; por Vía Pública con un 8,6\%; Radios con un 6,8\%; On-Line con un 6,2\%; Televisión de Pago con 
un 4,6; Revistas y Cine con un 1,9\% y 0,3\% respectivamente. En consecuencia, la inversión publicitaria en medios presenta una alta concentración en torno a dos medios: televisión abierta y prensa/diarios.

Esta concentración se explica, en parte, por la alta penetración de la televisión abierta y el tiempo destinado diariamente a su consumo; y en el caso de los diarios, su valoración en términos publicitarios se relaciona a su capacidad para congregargrandes audiencias de acuerdo con los diversos indicadores de lectoría y, por otra parte, por su influencia en la construcción de la agenda informativa. El diario no solo tiene la capacidad de tematizar la discusión social, sino que también de generar el debate que permita articular una opinión pública informada. Esta capacidad no debe entenderse de forma independiente de su dimensión de medio publicitario (Vergara, Santibáñez y Garrido, 2011).

\section{Inversión por medios (MM\$) Periodo 2011}

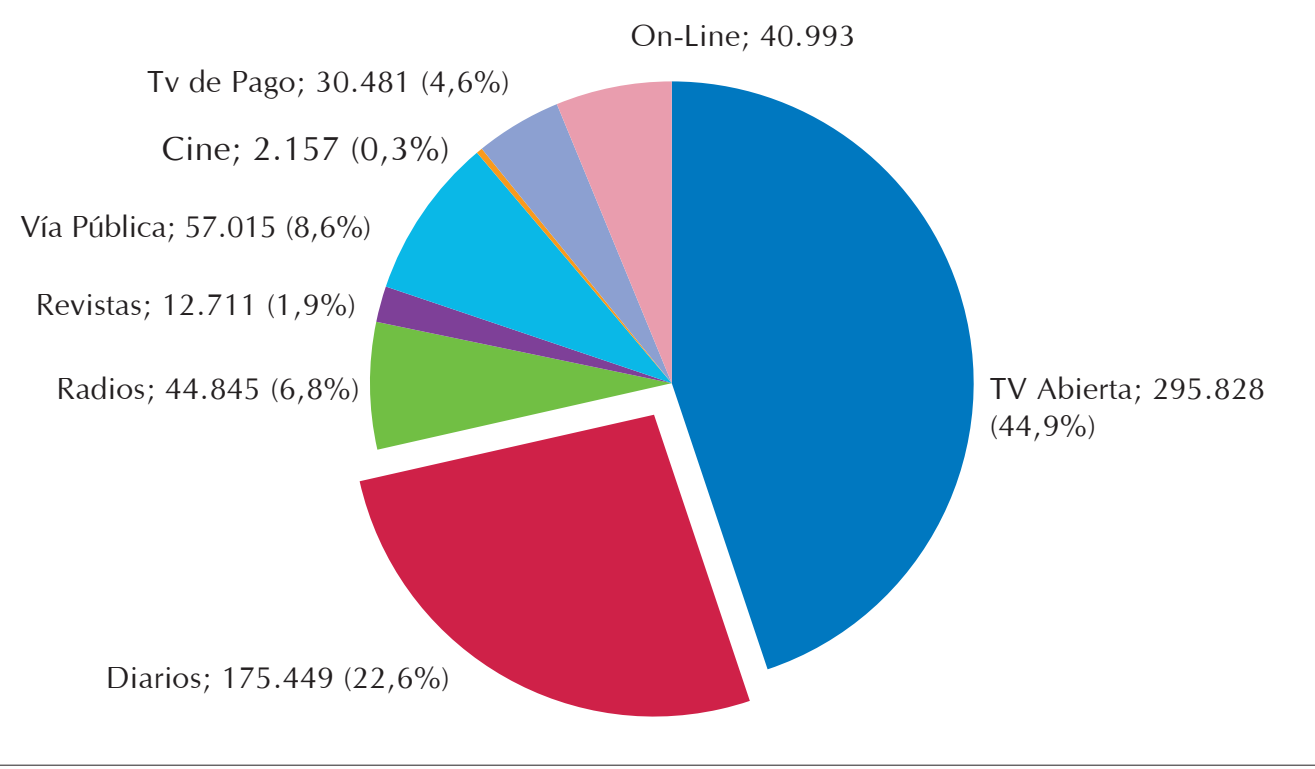

Fuente: Achap 2012

En la inversión publicitaria en Diarios, se advierte una fuerte concentración en torno a los dos grupos de comunicaciones que a su vez, presentan una alta concentración en la propiedad. Es así como el grupo El Mercurio, propietarios de los diarios El Mercurio, Las Últimas Noticias y La Segunda en Santiago, se estima que alcanza un $47,8 \%$ del total de la inversión. El grupo Copesa, propietario de los diarios La Tercera, La Cuarta y el diario gratuito La Hora, alcanza una inversión estimada de un 32,2\%. Ambos grupos suman un $80 \%$ de la inversión publicitaria 
en diarios. Junto a estas características generales, es importante considerar los cambios de formato que ha experimentado la publicidad en los últimos años a través de una hibridación de estos, rebasando los tradicionales límites del aviso publicitario.

\section{Avisaje información, pluralismo y definición de lo público}

Analizar la relación entre inversión publicitaria y las dinámicas informativas de los medios de prensa de acuerdo con el objetivo de este trabajo, requiere necesariamente de una aproximación metodológica que permita construir una línea de investigación que monitoree el fenómeno del pluralismo y el impacto de este en la presencia/ausencia de distintos avisadores considerados como relevantes, en términos de su comportamiento de inversión. Para esto, se diseñó una propuesta que permitiera incorporar dentro de nuestro análisis empresas anunciantes enfrentadas a situaciones de crisis, y por consiguiente, expuestas a la crítica pública.

\subsection{Consideraciones metodológicas de la investigación}

En primer lugar, debemos advertir que los datos obtenidos durante la etapa de trabajo de campo no constituyen una muestra estadísticamente representativa del tratamiento noticioso de los medios estudiados. Los datos obtenidos representan la cobertura informativa de cuatro casos específicos en un periodo acotado, por lo que su interpretación se circunscribe solo a este contexto.

El estudio realizado consideró la definición de dos unidades de análisis complementarias, las cuales en su conjunto permitieron dimensionar el efecto de los modelos de financiamiento en el tratamiento noticioso en términos de cobertura.

En primer lugar, se determinó cómo y con qué frecuencia se informan/cubren noticias que involucran a avisadores en situaciones de crisis. En segundo lugar, se determinó la perspectiva de distintos actores involucrados en el actual contexto medial, a nivel de medios de prensa escrita, agencias de comunicación estratégica, académicos del mundo de la comunicación y dirigentes del Colegio de Periodistas. En ambas etapas se consideraron los siguientes elementos base: selección de medios con modelos de financiamiento de alta y baja inversión publicitaria; selección y seguimiento de casos de avisadores con presencia de inversión publicitaria que estuviesen expuestos a situación de crisis; análisis de cobertura noticiosa y entrevistas en profundidad a actores de la industria medial.

Se analizaron los diarios El Mercurio, La Tercera y La Nación, entendidos los primeros dos como prensa de alta inversión y el tercero (reducido al momento del levantamiento de datos a formato electrónico), como medio de financiamiento mixto subvencionado mayoritariamente por el aporte estatal.

Posteriormente, se seleccionaron casos de notoriedad pública relacionados con avisadores 
en situación de crisis, a saber: Farmacias Ahumada, Salcobrand y Cruz Verde (colusión); línea aérea: LAN Cargo (conflictos laborales); Inmobiliaria Paz (demandas por publicidad engañosa) y alimentos Agrosuper (conflictos laborales y medio ambientales). Establecido este marco de análisis, se procedió al monitoreo durante tres meses de la cobertura dada a los anteriores casos en cada uno de los medios seleccionados, análisis que se complementó con la realización de un total de 10 entrevistas en profundidad a actores de la industria.

\subsection{Resultados y y discusión}

Durante los tres meses evaluados encontramos un total de 77 noticias relacionadas a los distintos casos analizados, con una participación diferenciada según medios en función de la inversión publicitaria, como puede observarse en el siguiente gráfico:

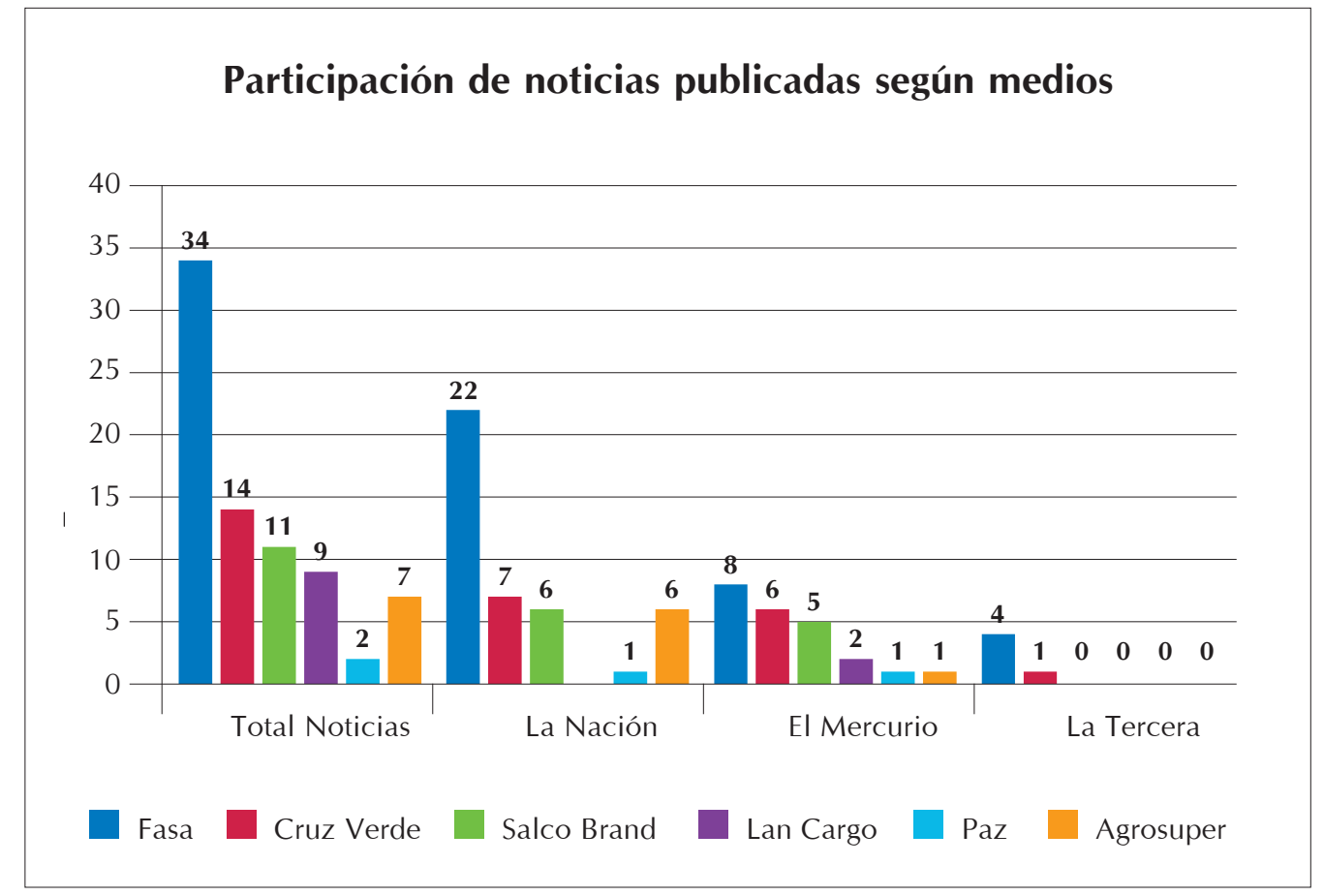

Fuente: Elaboración propia

Las cifras obtenidas muestran importantes diferencias en términos de la presencia e importancia de las empresas en situación de conflicto y el tratamiento dado a estas como hecho noticioso en los distintos medios evaluados. En este contexto destaca el papel de La Nación como productor de contenidos y cobertura, así como la diferencia respecto de medios que tradicionalmente concentran una alta inversión publicitaria. Si bien el modelo de negocios de 
La Nación descansaba en su dependencia de subsidio gubernamental (proveniente del Diario Oficial, que editaba la misma empresa), se destaca, en términos de la cobertura noticiosa, una mayor apertura al debate que considera cualitativamente la presencia de distintos actores como fuentes articuladores de una discusión pública.

Desde la perspectiva de las entrevistas realizadas a los actores de la industria, la relación entre cobertura noticiosa e inversión publicitaria se expresa en términos de una tensión que adquiere dos estados diferenciados. En primer término, aquellos que asumen esta realidad como manifiesta, la cual se expresa en la oferta comercial del medio al avisador, y en segundo término, como una expresión sutil en la práctica periodística diaria, en la que actúa la línea editorial del medio y su posición sociopolítica como condicionante del quehacer periodístico. Esto es, una especie de off implícito respecto del tratamiento de ciertos temas. Esta tensión puede abordarse analíticamente en función de dos dimensiones: por una parte, la propiamente comercial, y por otra, como la existencia de un alineamiento ideológico del medio. Ambas dimensiones impactan de manera directa, según los entrevistados, en la generación de la noticia y la cobertura asociada a esta. Esta segunda dimensión adquiere principal notoriedad en medios que han experimentado un crecimiento de su plataforma comercial y han desarrollado productos editoriales ad hoca la promoción de productos o servicios en un formato noticioso, como por ejemplo, publirreportajes. En estos medios, desde la perspectiva de los entrevistados, la plataforma comercial adquiere influencia sobre los productos editoriales al "ofrecer" como parte de la oferta de avisaje, un tratamiento diferenciado del avisador más allá de lo estrictamente publicitario. Si bien esto no constituye una práctica formal, sí sería parte de los argumentos de venta del medio, tensionando internamente la relación entre sus plataformas comerciales y sus departamentos editoriales, en un contexto en el que el imaginario periodístico habla de una "no influencia directa", pero existente. Cabe destacar que este tratamiento noticioso a un determinado avisador se vería acentuado por la sintonía ideológica del medio con el anunciante, lo que se expresaría a través de una agenda favorable al papel del avisador en el contexto país. En términos prácticos, el tratamiento diferenciado conllevaría el abordaje del avisador como contenido noticioso, expresándose en la presencia de notas sobre el anunciante más allá de la situación de crisis. Este efecto se potenciaría por la existencia de una plataforma comercial fuerte dentro del medio, la que ejercería una fuerte injerencia en lo editorial. A esto se suma, en algunos casos, el trabajo de las agencias de comunicación estratégica a través de una pre producción de productos editoriales.

A través de las entrevistas realizadas es posible identificar la promoción, por parte de algunos medios, de una visión ideológica pro empresa, visión que es reconocida a nivel de la sociedad y aceptada por los propios periodistas, lo que se traduce en un tratamiento "cuidadoso" del avisador. La existencia de este sub texto o escenario ideológico del medio desplaza la tensión hacia la práctica cotidiana del periodista, condicionando aspectos prácticos del oficio como el tipo de cobertura a realizar, el tratamiento de las fuentes, así como los tipos y tonos de la noticia.

Enlapráctica,sibien se reconoce la existencia de presiones por parte de avisadores o agencias 
de comunicación, estas se focalizarían en el área comercial, no articulándose en una influencia directa y verificable sobre la editorial del medio. En palabras de uno delos entrevistados: “... más que parar es moderar ( ... ), la noticia se da, el cuidado se da en el enfoque..... En este contexto, el tratamiento diferenciado del avisador en situación de crisis, estaría dado fundamentalmente por la existencia de redes de apoyo pro empresa y su reputación corporativa, más allá de su peso específico en el financiamiento del medio por la vía de la inversión publicitaria.

Si bien los medios autodefinen en términos imaginarios sus productos editoriales como independiente de la esfera comercial, la existencia de un alineamiento ideológico en situaciones de crisis del avisador, se traduce en requerimientos de doble cobertura y corrección de estilo del reportaje por parte del editor. El oficio del periodista en este escenario pasa necesariamente por entender la línea ideológica-editorial del medio y adecuarse a ese contexto: "[...] uno sabe en dónde está y qué cosas se pueden decir y cuáles no”.

Lo anterior adquiere especial complejidad al analizar los objetivos de gestión que realizan las agencias de comunicación. Estas, si bien reconocen la imposibilidad de influir directamente en los medios, buscan mediante las redes de contacto y de una relación histórica de avisaje en los medios, desarrollar labores de contención que garanticen la existencia o presencia mediática de la visión del avisador. Lo estratégico estaría dado por la capacidad para influir en el tratamiento noticioso del quehacer de la empresa más allá de las situaciones de crisis. Aspecto clave dentro de las metas de posicionamiento corporativo del avisador.

Si bien, como se ha señalado, la presión del avisaje no se manifesta directamente, esta se daría en el cruce entre tres factores. En primer lugar, a través del peso de la editorial y de su capacidad para crear un imaginario de neutralidad respecto del hacer periodístico; en segundo lugar, a partir del desarrollo de una plataforma comercial que condiciona de manera informal la cobertura y tratamiento noticioso, la que se alinea a nivel ideológico entre el medio y el avisador; y finalmente, en una cobertura asociada al avisaje como elemento de negociación, lo que se valida en el caso de las agencias de comunicación, como una práctica habitual. La interacción entre estos factores, implica que la independencia entre las dimensiones editorial y comercial del medio no se subordina necesariamente a la lógica del avisaje, sino que a la exposición de la marca en su dimensión política y pública. Esto confiere una dimensión política al avisador en su conexión con el medio, donde el avisaje agregaría una formalidad y complejidad al modus operandi del ejercicio periodístico.

No obstante lo anterior y por tratarse de prácticas informales, no es posible establecer el alcance real de la eventual intervención de los anunciantes en la cobertura de determinados contenidos. Esta "mediación” no se expresa de manera directa, sino que se asume en su dimensión práctica: en palabras de un entrevistado, “... lo que todos saben pero nadie ha visto...”. 


\section{Reflexiones finales}

Tanto la publicidad como el periodismo pueden ser considerados como una necesidad de la modernidad $y$, a la vez, parte constitutiva de la sociedad democrática contemporánea. ¿Están eximidos entonces de consecuencias negativas? A la luz de los resultados expuestos anteriormente, en absoluto.

Sin duda, nos encontramos ante un fenómeno de alta complejidad que mezcla dimensiones políticas, comerciales y de práctica profesional, impactando la producción de contenidos y la generación de un espacio de discusión pública. Esta posible "promiscuidad" entre el periodismo y la publicidad conlleva la presencia de ciertos riesgos que necesitan ser enunciados a fin de estimularla reflexión yel debate de los distintos actores involucrados. En efecto, y de acuerdo con lo expuesto, uno de los posibles riesgos para los medios es privilegiar una cobertura de carácter exclusivamente noticiosa, donde lo periodístico se reduce a lo estrictamente informativo y deja de ser un articulador de la discusión de lo público, donde por defecto se excluye determinados actores sociales a fin de aminorar posibles conflictos de interés a nivel editorial. La existencia de un Offen la relación con los avisadores, puede condicionar la cobertura a lo noticioso y no hacia la generación de una discusión frente al hecho producido, lo que afecta la generación de opiniones y de un mayor pluralismo en la información.

Lo anterior, pone de manifiesto la necesidad de replantear la ética de las comunicaciones de forma integrada y no acotarla solo a lo estrictamente periodístico o publicitario: es de gran importancia reconocer y abordar las zonas de contacto -y superposición- entre ambas actividades.

En este sentido, parece pertinente $-\mathrm{y}$ a la vez vigente- lo planteado por la Comisión Hutchins $^{3}$ respecto de la responsabilidad social de los medios y los protagonistas del proceso informativo (periodistas, medios, agencias de publicidad, consultoras de comunicación y avisadores), en términos de la necesidad de pensar la ética de la comunicación en términos más amplios. Es así como en el año 1947, en medio de una ola de reflexión innovadora que se produjo luego del final de la II Guerra Mundial, esta Comisión culminó su trabajo con un informe titulado "Una prensa libre y responsable", donde presididos por Robert M. Hutchins, Canciller de la Universidad de Chicago, fueron convocados once integrantes: profesores de derecho, filosofía, religión y economía y ningún periodista. La primera frase del informe es rotunda: "La Comisión se estableció para responder la pregunta de si 'la libertad de prensa está en peligro.' Nuestra respuesta es: 'Sí", la razón, se explicaba de inmediato, es que la prensa no ha "entregado un servicio adecuado a las necesidades de la sociedad". La prensa, decían también los miembros de la Comisión, se ha involucrado en prácticas que la sociedad condena. Este

3 El texto completo del informe se encuentra en:

http://ia700304.us.archive.org/22/items/freeandresponsib029216mbp/freeandresponsib029216mbp. pdf 
diagnóstico, pese al tiempo transcurrido y a los profundos cambios comunicacionales que hemos vivido, sigue siendo esencialmente correcto.

Desde la época del Informe Hutchins el mundo se ha expandido, pero especialmente en América Latina y en Chile, la convicción de que no basta con la dictación de leyes para asegurar el buen manejo de los medios. Se requiere un ejercicio éticamente responsable de la libertad de expresión.

Un sector académico significativo, liderado en América Latina por el colombiano Javier Darío Restrepo (2008), ha insistido en que se debe reforzar la autorregulación como la única manera de proteger la libertad de expresión. Sobre esta base, se han formulado códigos y recomendaciones en materia ética que no se imponen porla ley,perotienen un imperativo moral generalmente aceptado y reconocido. Lo mismo ha ocurrido en materia de autorregulación publicitaria. Pero este panorama es todavía insuficiente, requiere una visión de conjunto que abarque al resto de los actores del proceso comunicacional. El desarrollo de la industria de los medios de comunicación, la revolución tecnológica y la consolidación en prácticamente todo el mundo del modelo de libertad económica, genera situaciones que obligan a revisar conceptos naturalizados.

En un esfuerzo por resumir el desafío que implica para periodistas, responsables de medios y avisadores superar las tentaciones que pueden afectar la información, Louis Day ha señalado:

“...los intereses comerciales pueden afectar la función periodística a través de la presión directa sobre los editores y otros directivos de los medios. Los avisadores generalmente se molestan cuando son objeto de informaciones que no los favorecen y a veces reaccionan retirando sus avisos de las publicaciones o estaciones que les molestan o tratando de presionarlos para que supriman informaciones poco gratas. En otras ocasiones, los avisadores pueden intentar persuadir o manipular a los medios para que les den cobertura favorable en determinados casos. Estos tipos de presión plantean un difícil dilema ético para reporteros y editores cuyos salarios se pagan, en parte, gracias a los ingresos por publicidad. Sin embargo, la verdadera libertad de prensa en un sistema democrático requiere de periodistas que, en primer lugar, sean responsables ante la sociedad civil y estén al servicio del interés público. Es importante que las funciones periodística y comercial del medio permanezcan separadas y que las decisiones editoriales se tomen sin depender de influencias externas. De otro modo, la prensa se subordina a los intereses económicos especiales y pierde relevancia (y, por tanto, influencia) en el proceso democrático" (2001, p. 38). 


\section{Referencias bibliográficas}

Asociación Chilena de Agencias de Publicidad ACHAP. (2012). Inversión publicitaria 2011. Extraído el 21 de noviembre de 2012, desde http://www.achap.cl/documentos/ Inversion2011.pdf.

Brunner, J. y Catalán, C. (1995). Televisión, libertad, mercado y moral. Santiago de Chile: Los Andes.

Bustamante, E. (1994). La concentración en la comunicación y la cultura. Barcelona: Ponencia en Converses a la Pedrera.

Catalán, C. (1982). El mercado de revistas de actualidad y la inversión publicitaria: el caso de Chile. Documento de Trabajo ILET DEC/D/89.

Corrales, O. y Saldoval, J. (2003). Concentración del mercado de los medios, pluralismo y libertad de expresión. Extraído el 8 de agosto de 2012, desde http:/ / www.comunicacion. uchile.cl/docs/corrales2005.pdf.

Day, L. (2001). La tradición democrática. En Castellón, L. (Ed.), La ética periodística en el nuevo milenio. Santiago de Chile: Cuarto Propio.

Habermas, J. (1981). Historia y crítica de la opinión pública: la transformación estructural de la opinión pública. Barcelona: Gustavo Gili.

Habermas, J. (1987). Teoría de la acción comunicativa. Racionalidad de la acción y racionalización social. Madrid: Taurus.

Janus, N. y Rocagliolo, R. (1987). Publicidad, medios de comunicación y dependencia. En Publicidad la otra cultura. Cristianismo y comunicación en América Latina. Lima: IPAL.

Lazarsfeld, P. (1985). La campaña electoral ha terminado. En Miquel de Moragas (Ed.), Sociología de la comunicación de masas III: Propaganda política y opinión pública. Barcelona: Editorial Gustavo Gili.

Moragas de M. (1985). Política y sociología en la comunicación de masas. En Sociología de la comunicación de masas III: Propaganda política y opinión pública. Barcelona: Gustavo Gili.

Llorens, C. (2001). Concentración de empresas de comunicación y pluralismo: la acción de la UE. Tesis para optar al grado de Doctorado en Comunicación. Universidad Autónoma de Barcelona, Barcelona.

Madrid, S. (2005). Semiótica del discurso publicitario. Del signo a la imagen. Murcia: Publicaciones de la Universidad de Murcia.

Pérez-Tornero, J. M. (1991). Para una toma de conciencia del fenómeno publicitario. En Mattelart, A. (Ed.), La publicidad. Barcelona: Paidós.

Portales, C. (1999). La concentración de los medios y la libertad de expresión en Chile. Documento de Trabajo. Universidad de Chile.

Restrepo, J. (2008). La niebla y la brújula. Bogotá: Random House Mondadori. 
Sontag, S. (2001). El afiche: publicidad, arte instrumento político, mercancía. En Fundamentos del diseño gráfico. Buenos Aires: Ediciones Infinito.

Sánchez Tabernero, A. (2002). Concentración de las empresas de comunicación en Europa: los nuevos datos contradicen los viejos mitos. Comunicación y Sociedad, 15 (1).

Santibáñez, A. y Vergara, E. (2008). Periodismo y Publicidad: claves y ambigüedades de una relación promiscua. Universum, Revista de Humanidades y Ciencias Sociales, 1 (23).

Sunkel, G. y Geoffroy, E. (2001). Concentración económica de medios de comunicación. Santiago de Chile: Lom Ediciones.

Thompson, J. (1998). Los media y la modernidad. Una teoría de los medios de comunicación. Barcelona: Paidós.

Vergara, E., Santibáñez, A. y Garrido, C. (2011). Pluralismo informativo y dependencia publicitaria. Una aproximación a los límites de la libertad de expresión. Barcelona: Ponencia al VI Congreso Internacional de Comunicación y Realidad. 